Cipango $\begin{aligned} & \text { Cipango } \\ & \text { Cahiers d'études japonaises }\end{aligned}$

15 | 2008

Guerre, colonialisme et commémoration

\title{
Tradition du mouvement dans les écoles classiques de combat du Japon
}

\section{Kacem Zoughari}

\section{OpenEdition}

1 Journals

\section{Édition électronique}

URL : https://journals.openedition.org/cipango/440

DOI : $10.4000 /$ cipango.440

ISSN : 2260-7706

\section{Éditeur}

INALCO

\section{Édition imprimée}

Date de publication : 1 janvier 2008

Pagination : 267-273

ISBN : 978-2-85831-177-4

ISSN : $1164-5857$

\section{Référence électronique}

Kacem Zoughari, «Tradition du mouvement dans les écoles classiques de combat du Japon », Cipango [En ligne], 15 | 2008, mis en ligne le 14 novembre 2011, consulté le 30 juin 2021. URL : http:// journals.openedition.org/cipango/440 ; DOI : https://doi.org/10.4000/cipango.440

Ce document a été généré automatiquement le 30 juin 2021.

\section{(c) (7) (8)}

Cipango est mis à disposition selon les termes de la Licence Creative Commons Attribution - Pas d'Utilisation Commerciale 4.0 International. 


\section{Tradition du mouvement dans les écoles classiques de combat du Japon}

Kacem Zoughari

\section{RÉFÉRENCE}

Tradition du mouvement dans les écoles classiques de combat du Japon, Thèse de doctorat soutenue par Kacem Zoughari (Inalco, 2008), sous la direction de F. Macé et J.-N. Robert.

1 «Les chemins de la patience croiseront ceux de l'audace. Un jour ou l'autre viendra bien le moment de se décider, nous sommes-nous souvent répété. Les exercices lents, en apparence répétitifs, que suppose l'acquisition des fondamentales, nous entraîneront vers des régions au relief plus accidenté, où les sentiers courent selon un tracé imprévu et parfois cessent d'êtres balisés. Ces images, d'ailleurs, sont approximatives. Sans doute serait-il plus simple et plus juste de dire: le travail de l'enseignement implique de façon nécessaire un effort égal de recherche. L'un est le corollaire de l'autre. [...]»

2 Rédigées par J.-J. Origas pour l'introduction du premier volume de Cipango en 1991, ces quelques lignes montrent merveilleusement les différentes étapes qu'il faut franchir dans la rédaction d'une thèse dont le sujet principal concerne les techniques de combat, communément nommées arts martiaux. Les études sérieuses réalisées sur cette question en français sont rares. Mis à part le remarquable travail de Kenji Tokitsu, la seule étude sérieuse est celle de François Roger (Inalco, 1994) sous la direction de Francine Hérail.

3 Il est important de souligner que les études sur les arts martiaux sont parfois dépréciées par les enseignants et les chercheurs. Pour un grand nombre de personnes, chercheurs et enseignants, les "arts martiaux» désignent les sports de combat qui incluent les disciplines, mondialement connues pour certaines, comme le jūdō 柔道, le 
kendō剣道, le karate 空手, l'aikidō 合気道, le iaidō 居合道, etc., et englobent également d'autres disciplines provenant généralement d'Asie. Or, l'analyse étymologique de l'expression « arts martiaux » montre qu'à l'origine, il ne s'agit pas de sports. En effet, les « arts martiaux » désignent fondamentalement toutes les techniques utilisées sur un champ de bataille, pour un combat dont l'issue est la survie. Il inclut à la fois des notions de stratégie, de tactique, de combat sous toutes ses formes, d'utilisation des armes les plus diverses, de la connaissance du terrain, de la psychologie, de la survie, etc. Il existe d'ailleurs un grand nombre de termes en japonais qui véhiculent la notion de «pratique d'arme».

Dans mon étude, j'utilise le terme inscrit dans les plus vieux documents de transmission, heihō ou hyōhō 兵法, qui désigne toutes les techniques de combat, stratégies et tactiques militaires mises en œuvre pour survivre sur le champ de bataille, devancer le danger et l'anéantir avant qu'il ne se déclare. Le terme « arts martiaux » en lui-même n'est pas si éloigné des différents aspects désignés par le heihō. En d'autres termes, l'expression « arts martiaux » ne peut désigner des disciplines sportives mises au point pour l'éducation physique. C'est pour cette raison que j'ai utilisé l'expression " école de combat » dans le titre de ma thèse plutôt que celui d'" arts martiaux ", ceci afin d'éviter tout amalgame.

5 L'hypothèse principale qui guide mon étude est que la kinésiologie et l'ergonomie de la préparation au combat effectif (avec des armes et des armures réelles) reposent sur des principes radicalement différents de ceux qui ont servi à formaliser la grammaire du kendō, du iaidō, du karate ou du jūdō. Effectivement, l'analyse de l'utilisation du corps dans les disciplines créées durant l'ère Meiji montre que l'essentiel des mouvements et des techniques du corps prennent leur départ à partir de l'utilisation des hanches.

Dans le cas des disciplines permettant l'utilisation des armes comme le kendō ou le iaidō, on constate chez la plupart des pratiquants le développement d'un antagonisme corporel qui provoque un déséquilibre physique et une réelle limitation quant à l'utilisation de son corps. J'ajoute que ces disciplines obéissent à des critères bien précis qui sont propres à ceux de l'éducation physique et sportive et non à ceux nécessaires à "la survie en combat». C'est de là que découle un grand nombre de blessures caractéristiques à ces pratiques. Sans oublier ces "réelles limitations" quant à l'utilisation de son corps et au maniement de l'arme du fait que, dans ces disciplines, le nombre de techniques est très limité.

7 En revanche, les écoles classiques proposent, lorsque la pratique n'est pas figée autour du kata 型, «forme préétablie d'une séquence de mouvement matérialisant une technique ", un très large éventail de techniques de combat et d'armes articulées autour d'une utilisation du corps bien spécifique. Cette utilisation du corps découle directement de l'idéologie du combat qui avait cours durant la période féodale au Japon, c'est-à-dire de pouvoir utiliser différentes armes à partir d'une seule position, d'une seule utilisation du corps. C'est de là que réside le point de départ de ma recherche.

8 Quel est le processus de pratique, la forme du corps, du mouvement qui permet à un maître âgé de pouvoir encore pratiquer et d'atteindre un niveau lui permettant d'utiliser son corps plus efficacement qu'un adepte des disciplines modernes se trouvant dans la force de l'âge? Quelles sont les caractéristiques de l'attitude corporelle de base et du processus de pratique qui permettent d'atteindre une utilisation aussi subtile du corps dont l'économie de mouvement représente le cœur? 
Comment trouver le point commun entre la calligraphie, le dessin, la danse, le théâtre, la cérémonie du thé, qui se trouve dans une certaine utilisation du corps issue de la pratique des armes et de la survie en combat?

9 Pour pouvoir comprendre comment un maître fondateur arrivait à atteindre ce niveau subtil de maitrise du corps et pouvait l'appliquer dans sa vie de tous les jours, j'ai, en premier lieu, étudié minutieusement toutes les informations historiques sur les maîtres fondateurs et sur leurs successeurs les plus connus de la première partie de la période d'Edo.

10 Grâce à cette étude, j'ai pu isoler un grand nombre de points communs, comme : l'âge avancé dans la pratique et le maintien d'un conditionnement physique et psychique (débouchant sur une certaine utilisation du corps permettant de vaincre aisément de plus jeunes guerriers expérimentés...), la connaissance et la maîtrise de diverses armes, l'application à diverses disciplines, etc.

11 J'ai également élargi mes investigations aux différents documents de transmission rédigés par plusieurs maîtres afin de trouver la première trace écrite décrivant ce processus de pratique et expliquant la forme corporelle de départ permettant d'atteindre ce niveau d'excellence.

12 Mon choix s'est porté directement sur l'école Shinkage-ryū 新陰流fondée par Kamiizumi Ise no kami Hidetsuna (1508-1582). En effet, cette école propose la première source d'information historique permettant non seulement de comprendre profondément les différents modes de transmission qui avaient cours dans les trois premiers courants du Japon fondés durant la période Muromachi, mais permet aussi d'avoir une image précise de l'attitude corporelle de base et du processus de pratique d'un maître fondateur de haut niveau vivant entre les périodes Muromachi (1336-1573) et Edo (1603-1868).

13 Les plus vieux documents de transmission, dont l'authenticité est reconnue de tous les chercheurs japonais en "budōlogie », ont été rédigés par Hidetsuna, Yagyū Muneyoshi

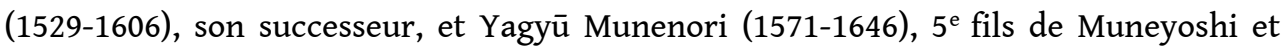
instructeur de la famille Tokugawa. Ils ont été transmis jusqu'à nos jours et nous avons pu avoir accès non seulement aux originaux, mais aussi aux différents documents de transmission, chroniques, notes et lettres relatives à la pratique et la transmission du savoir de cette école qui s'étalent sur plus de 20 générations. J'ai donc choisi les premiers documents de transmission, car ils permettent, entre autres, de cerner et de comprendre précisément les différentes caractéristiques de l'attitude corporelle et de l'utilisation du corps entre les périodes Muromachi (1336-1573), Momoyama-Azuchi (1573-1598) et Edo (1603-1868).

14 J'ai donc divisé mon étude en trois parties dont le fil conducteur est la relation de maitre à disciple de laquelle dépend la transmission des techniques de combat et des documents rédigés par le maître.

15 Dans la première partie intitulée "Les Maîtres ", je présente toutes les informations historiques relatives aux trois premières générations du Shinkage-ryū. Il s'agit principalement du cadre historique dans lequel le Shinkage-ryū a été fondé et comment il fut transmis. Y est présenté le plus précisément possible le parcours initiatique, les rencontres, les batailles et combat, qui vont pousser nos trois personnages principaux à prendre la voie des armes. Pour faciliter la compréhension, j'ai ajouté une présentation 
générale des différents aspects de la fondation et de la transmission des premiers ryū (écoles anciennes) qui étaient à l'origine du Shinkage-ryū.

Dans la seconde partie, intitulée "Les documents de transmissions", je présente les trois documents de transmissions les plus importants du Shinkage-ryū: le Kagemokuroku 影目録, rédigé en 1566, le Shinkage-ryū Heihō mokuroku no koto 新陰流兵法 目 録事, rédigé en 1601, et le Heihō Kaden-sho 兵法家伝書, rédigé en 1632. Dans cette partie, plutôt que de me limiter à une traduction littérale, j'ai opté pour une analyse des caractéristiques principales de ces trois documents qui sont la transmission du savoir, c'est-à-dire l'attitude corporelle de départ de laquelle découle la subtilité découverte par le maître, et le processus de conditionnement physique et psychique qu'il faut maintenir durant toute la vie.

Il convenait ici de suivre la pensée des différents auteurs qui reflétait une pratique basée sur l'expérience vécue. C'est selon cette perspective que j'ai traduit la plupart des termes qui paraissaient ambigus en me référant sans cesse à l'application et aux démonstrations auxquelles j'ai pris part. Il fallait constamment privilégier une analyse en rapport avec l'utilisation du corps, le maniement des armes, le combat et ses différents aspects psychologiques et physiques.

La grande difficulté de cette partie résidait principalement dans la compréhension des instructions et explications liées à l'utilisation du corps, dont la réelle signification était le plus souvent dissimulée derrière des termes techniques empruntés, entre autres, au bouddhisme ou au confucianisme. Dans ce cas également, j'ai privilégié la pratique et l'application pour comprendre les termes.

L'analyse et les explications relatives à ces trois documents m'a permis de présenter dans la dernière étape de mon étude la structure et la forme du mouvement qui forment la base de départ du processus de conditionnement physique et psychique d'où découle l'ultime subtilité permettant une utilisation du corps bien spécifique.

Dès le début de la rédaction de cette troisième partie, il était évident que la seule traduction des explications rédigées n'était pas suffisante pour comprendre l'utilisation du corps d'un maître. Il fallait trouver le point de départ qui permettait à ce dernier de combattre sans mouvement inutile, de grand déploiement musculaire et ainsi déboucher sur une utilisation du corps où souplesse et économie de mouvement étaient les principales caractéristiques. Dans cette partie, j'ai opté pour une présentation des données et instructions les plus complètes afin de pouvoir les analyser et d'en offrir une image plus précise.

21 Le point de départ était de comprendre les contraintes liées au port de l'armure, de pouvoir les sentir et de recréer les mêmes sensations. Une fois cette étape de la recherche réalisée, on peut enfin définir plus précisément l'attitude corporelle de départ mise au point par Hidetsuna. Celle-ci avait été dessinée par Hidetsuna et Muneyoshi dans leurs documents, mais il était aussi essentiel de comprendre les raisons de cette attitude et les conditions qu'elle imposa à la tenue du corps et à son utilisation.

La maîtrise corporelle nécessaire pour arriver à tenir cette attitude avec et sans armure montre que sa transmission était très difficile. Effectivement, cette attitude corporelle ne pouvait être comprise qu'à un moment bien précis du combat, celui où l'arme ennemie va entrer en contact avec une partie du corps. C'est à ce moment-là qu'il fallait adopter cette attitude en un seul mouvement, une seule rotation afin de pouvoir 
esquiver l'attaque et se placer de telle façon à être parallèle à l'arme de l'ennemi, en d'autres termes, se trouver sur le fil de la lame.

Une fois les caractéristiques et contraintes de cette attitude corporelle de départ analysées, l'autre aspect de cette partie consiste à montrer comment un maître fondateur appliquait la subtilité issue de cette maitrise du corps à différents aspects de sa vie quotidienne qui pouvaient offrir, en ces périodes de guerres, de multiples occasions à d'éventuels ennemis de pouvoir s'en prendre à lui. C'est ainsi que j'ai présenté deux aspects essentiels qui sont «l'art de se mettre debout» à partir de la position assise et « l'art de marcher».

24 Savoir s'asseoir de manière à pouvoir utiliser une arme ou contrer une attaque tout en étant assis, tout comme se lever sans ne manifester aucun signe d'attaque ou de défense, est un art. En effet, le simple fait de pouvoir utiliser le corps dans sa totalité en un mouvement continu requiert une profonde maîtrise du corps et des autres facteurs que sont la distance et l'espace. C'est cette maîtrise que l'on retrouve dans l'attitude corporelle de départ qui devait être appliquée aux différentes actions de la vie quotidienne. Pour cet aspect de l'étude du mouvement, en plus des rencontres et de la pratique avec différents maîtres, j'ai pu rencontrer des spécialistes comme Yumio Nawa (1912-2006) qui expliquaient les us et coutumes des guerriers aux acteurs de cinéma et de feuilletons télévisés qui avaient des rôles de guerrier.

Le dernier aspect de cette étude est la présentation et l'analyse de la marche. Celle-ci représente le niveau ultime d'un maitre, car il n'y a plus de forme de combat, tout doit être dissimulé, caché, invisible, tout en permettant de faire face à tout type d'attaque. Pour celle-ci également j'ai expérimenté différents types de marches et analysé les explications des documents de transmissions afin d'en donner une image suffisamment précise.

26 Enfin, dans la conclusion de cette étude, je présente en quatre points les aspects essentiels liés à la pratique et à la transmission des techniques de combat dans les écoles classiques du Japon. Certains de ces aspects sont encore appliqués avec des certaines modifications jusqu'à nos jours.

27 Le premier est le cadre de la relation maître à disciple et la signification du terme " maître fondateur » que j'ai utilisé tout au long de ma thèse. Le second point est la nature du document de transmission, ce qu'il doit permettre et quelles sont les clefs nécessaires pour comprendre et interpréter son sens. Le troisième point concerne les blessures, car la pratique des différents maîtres que j'ai présentés avait sûrement laissé quelques séquelles. Aussi comment avaient-ils pu les contourner? Enfin le quatrième point est la notion de mouvement régénérateur qui découle du conditionnement physique et psychique mis au point par les maîtres présentés. 